\title{
Boid-Inspired Harmony Search Approach to Aircraft Parameter Estimation*
}

\author{
Junnan Li \\ Science and Technology on Aircraft Control Laboratory \\ School of Automation Science and Electrical Engineering \\ Beihang University (BUAA) \\ Beijing100191, China \\ lijunnanbuaa@163.com
}

\author{
Haibin Duan ${ }^{1,2}$, Senior Member, IEEE \\ 1. Science and Technology on Aircraft Control Laboratory \\ Beihang University (BUAA), Beijing100191, China; \\ 2. Provincial Key Laboratory for Information Processing \\ Technology, Soochow University, Suzhou 215006, China \\ hbduan@buaa.edu.cn
}

\begin{abstract}
Aircraft parameter estimation is a vital technology in aeronautical industry. It is the fundamental of control law design and aircraft performance evaluation. Harmony Search (HS) is proposed in 2001, which mimics music players composing fantastic music. Its performance has been verified by many benchmark problems. In 1986, Reynolds proposed a mathematical model describing animals' group behavior, and he generally named the flocking creature "boids", which can be interpreted as "bird like objects". We modify the pitch adjustment step of HS inspired by the boid model. Our new method is named Boid-Inspired Harmony Search (BIHS). This paper investigates the application of BIHS to aircraft parameter estimation problem. In this work, the longitudinal state-space model of F-18 jet is used. It is shown that the BIHS approach is efficient, and in some ways, has an advantage over the classical Maximum Likelihood (ML) method.
\end{abstract}

Index Terms -aircraft, parameter estimation, Boid-inspired Harmony Search

\section{INTRODUCTION}

System identification is a well studied filed. Several classical methods, such as Least Square (LS) method and Maximum Likelihood method have been proposed in the past century. With the development of computer industry, scientists and engineers are able to process a much larger data set obtained from tests, which makes some effective methods possible to be implemented in practice.

Early research in system identification mainly focuses on several specific types of models. Nowadays, applications in different fields raise new challenges to identification technique [1-2]. Since 1960s, parameter estimation technique has been successfully applied to aircraft parameter estimation. As the fundamental of control law design and performance evaluation, it becomes a vital technology in aeronautical industry. The central issue in aircraft parameter estimation is to predict and evaluate the performance and dynamical characteristics of a flight vehicle [3]. The direct estimation methods can be roughly classified into three categories: 1) equation error method, 2) output error method, 3) filter error method. The indirect estimation method sets the unknown parameters as augmented states, and applies a filter to estimate the augmented state variables. The equation error and output error methods are deterministic methods, whereas the filter error method and the indirect methods are statistical [4-6].

More recently, new methods, such as neural network and intelligent optimization algorithms have been investigated to solve parameter estimation problems [7-10]. The research on intelligent optimization algorithm is a flourishing filed today [11-13]. Among them, bio-inspired algorithms mimic creatures' collective behavior to search for the global optimum of the cost function. Duo to their favourable statistical property, intelligent optimization algorithms are seen as a promising alternative method to traditional parameter estimation approaches. Genetic Algorithm (GA) is the first heuristic algorithm used in parameter estimation problems, and its capacity has been verified in many cases. In recent years, many novel optimization algorithms are proposed. Among them, HS is a promising one which has some advantages over GA. When generating a new individual, HS considers all the individuals in the group, while GA only considers two parents. So HS is supposed to utilize the global information of the group more adequately.

In this paper, we proposed a Boid-Inspired Harmony Search (BIHS) algoithm for solving the aircraft parameter estimation problems, and comparative results are also given to verify the feasiblity and effectiveness of our proposed BIHS approach.

\section{BOID-INSPIRED HARMONY SEARCH ALGORITHM}

\section{A. The Basic HS}

Inspired by musical performance, Z. W. Geem and J. H. Kim proposed HS in 2001[14].They realized the optimization process and musical performance have many common factors. They created a novel heuristic algorithm which searches for the global optimum imitating musicians compose fantastic music. Similar to other bio-inspired algorithms, such as GA, Particle Swarm Optimization, and Artificial Bee Colony, HS is also based on the concept of population. HS also has favourable statistical property. Its exploratory power has been proved in theory [15]. There are four key steps in HS. The flowchart of this basic HS algorithm is shown in Fig. 1.

\footnotetext{
* Natural Science Foundation of China (NSFC) under grant \#61333004 and \#61273054, Aeronautical Foundation of China under grant \#20135851042, Open Fund of the Provincial Key Laboratory for Information Processing Technology, Suzhou University under grant \#KJS1020,and Graduate Innovation Foundation for Beihang University under grant \#YCSJ-01-201405
} 


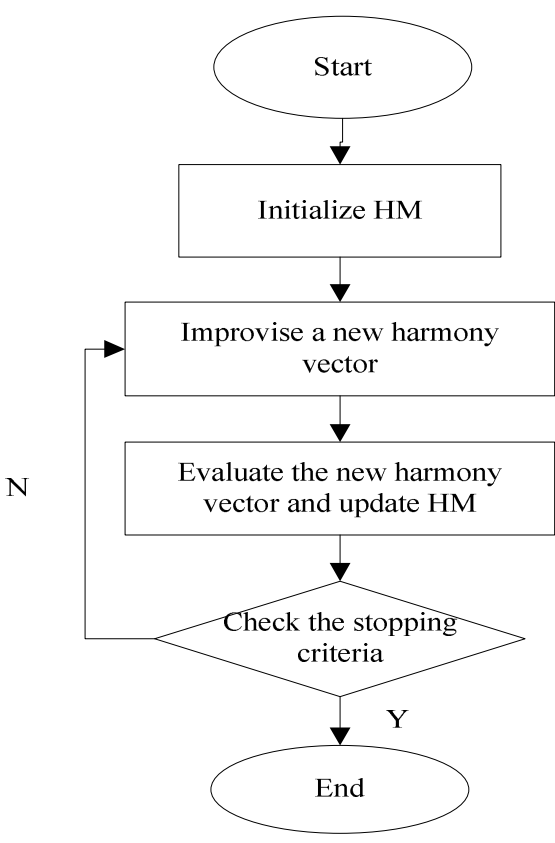

Fig.1. Flowchart of the basic HS

Step1: Initialize HM. Harmony memory (HM) mimics the memory in a musician's brain. HM contains a group of harmony vectors, each element in the vector represents a pitch. In this step, the algorithm generates a group of random vectors and stores them in HM. Each pitch is generated according to (1),

$$
x_{i}^{r}=x_{i}^{l}+\operatorname{rand} \times\left(x_{i}^{u}-x_{i}^{l}\right) \quad i=1 \ldots N
$$

where $x_{i}^{u}$ and $x_{i}^{l}$ are the upper and lower bounds of the $i$-th element respectively. See in (2).

$$
\mathrm{HM}=\left[\left(\begin{array}{c}
x_{1}^{1} \\
x_{1}^{2} \\
\vdots \\
x_{1}^{N}
\end{array}\right)\left(\begin{array}{c}
x_{2}^{1} \\
x_{2}^{2} \\
\vdots \\
x_{2}^{N}
\end{array}\right) \quad \ldots\left(\begin{array}{c}
x_{\mathrm{HMS}}^{1} \\
x_{\mathrm{HMS}}^{2} \\
\vdots \\
x_{\mathrm{HMS}}^{N}
\end{array}\right)\right]
$$

Step2: Improvise a new harmony vector. The algorithm uses harmony memory consider rate (HMCR), pitch adjustment rate (PAR), and searching step $b w$ to improvise a new harmony vector. Each element in the new vector is generated separately. For each element, the algorithm decides whether to generate a brand new one, or to choose an existing one from HM and adjust it. The improvising step is described as follows:

$$
\begin{aligned}
& \text { If rand }>\text { HMCR } \\
& \quad \mathrm{x}_{i}^{\text {new }}=x_{i}^{l}+\operatorname{rand} \times\left(x_{i}^{u}-x_{i}^{l}\right)
\end{aligned}
$$

Else, $x_{i}^{r}=x_{i}$

$$
\begin{aligned}
& \text { If rand }<\text { PAR } \\
& x_{i}^{\text {new }}=x_{i}^{r} \pm \text { rand } \times b w \\
& \text { Else, } \\
& x_{i}^{\text {new }}=x_{i}^{r}
\end{aligned}
$$

Step3: Update HM. In this step, the performance of the new vector is evaluated by the cost function, if it performs better than the worst vector in HM, then the worst vector will be replaced by the new one.

Step4: Check the stopping criteria. If the stopping criteria are satisfied, end the algorithm. Otherwise, go back to the improvisation step.

\section{B. The Improved $H S$}

The performance of HS has been verified by many benchmark problems; however, there is still room for improvement. If HMS is large, in the initialization of HM, there is a possibility that a vector in HM is near the global optimum solution. To accelerate the convergence rate of HS, the new improvised vector in Step2 should approach the best solution in HM more quickly. Some researchers improved the basic HS and created a new method called Global-best Harmony Search (GHS) [16]. In GHS, the pitch adjusting step is modified by

$$
x_{i}^{\text {new }}=x_{i}^{\text {best }}
$$

GHS does accelerate the convergence rate of the algorithm. However, people worry that the modified pitch adjusting step leads to premature convergence to local optimum. In this paper, we propose a novel method inspired by the boid model. The boid model was proposed by Reynolds in 1986. It consists of three simple steering behaviors: separation, alignment and cohesion [17]. In cohesion behavior, boids gather towards the center of the flock. In HS, we want each pitch to converge faster to the best pitch. So we introduce the cohesion behavior into the pitch adjusting step. The idea is that, the algorithm choose a pitch $x_{i}^{r}$ in HM, if the distance between $x_{i}^{r}$ and $x_{i}^{\text {best }}$ is large, $x_{i}^{r}$ approaches $x_{i}^{\text {best }}$ with a large velocity. If $x_{i}^{r}$ is near $x_{i}^{\text {best }}$, the algorithm adjust $x_{i}^{r}$ with the searching step $b w . x_{i}^{\text {best }}$ denotes the $i$-th element of the best harmony vector in HM. 


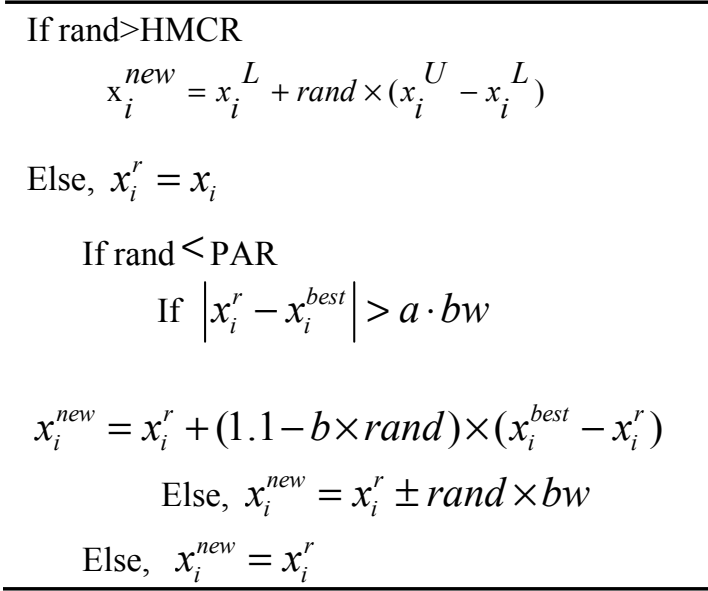

Parameters $a$ and $b$ affect the efficiency of the new algorithm. The selection of $a$ depends on how you define a large distance, the parameter $b$ affects the velocity. On the one hand, we expect our method to accelerate the convergence rate of HS; on the other hand, we try to enhance the randomness of the algorithm. In this paper, we set $a$ be equal to 10 , and $b$ be equal to 0.5 .

\section{System IDENTIFICATION PROBLEM}

Generally, a dynamic model for identification is described as follows:

$$
\begin{aligned}
\dot{x}(t) & =f[x(t), u(t), \theta]+F w(t), \quad x\left(t_{0}\right)=x_{0} \\
y(t) & =g[x(t), u(t), \theta] \\
z(k) & =y(k)+G v(k), \quad k \in N
\end{aligned}
$$

Equation (4) is the dynamic equation, (5) is the output equation, and (6) is the observation equation of the model. $w(t)$ denotes the process noise, and $v(k)$ denotes the measurement noise, usually, they are assumed to be zero-mean white Gaussian noise. Matrixes $F$ and $G$ are process noise matrix and measurement noise matrix respectively. $x_{0}$ is the initial state of the system. In fact, $F, G$ and $x_{0}$ can also be seen as unknown parameters to be estimated. However, in most cases, we are more interested in $\theta$, see in Ref. [6]. $\theta$ is written in the following form:

$$
\theta=\left[\theta^{1}, \theta^{2}, \cdots, \theta^{n}\right]^{\mathrm{T}}
$$

\section{A. Longitudinal State-space Model of F-18}

In this paper, we estimate the parameters lying in the longitudinal state-space model of F-18 via a novel evolutionary algorithm BIHS. The longitudinal small perturbation state-space model is as follows:

$$
A=\left[\begin{array}{cccc}
X_{V} & X_{\alpha} & X_{q} & X_{\theta} \\
Z_{V} & Z_{\alpha} & Z_{q} & 0 \\
M_{V} & M_{\alpha} & M_{q} & 0 \\
0 & 0 & 1 & 0
\end{array}\right] \quad B=\left[\begin{array}{cc}
X_{\delta_{e}} & X_{\delta_{T}} \\
Z_{\delta_{e}} & X_{\delta_{T}} \\
M_{\delta_{e}} & X_{\delta_{T}} \\
0 & 0
\end{array}\right]
$$

$$
\begin{aligned}
C & =\left[\begin{array}{llll}
1 & 0 & 0 & 0 \\
0 & 1 & 0 & 0 \\
0 & 0 & 1 & 0 \\
0 & 0 & 0 & 1
\end{array}\right] \\
X & =\left[\begin{array}{llll}
V & \alpha & q & \theta
\end{array}\right]^{\mathrm{T}} \quad u=\left[\begin{array}{ll}
\delta_{e} & \delta_{T}
\end{array}\right]^{\mathrm{T}}
\end{aligned}
$$

In this work, we try to estimate all the parameters in $A$ and partial parameters in $B$ pertaining to the elevator input. Therefore, $\theta$ is written as:

$$
\begin{aligned}
\theta= & {\left[X_{v}, X_{\alpha}, X_{q}, X_{\theta}, Z_{v}, Z_{\alpha}, Z_{q}, M_{v}, M_{\alpha}, M_{q},\right.} \\
& \left.X_{\delta_{e}}, Z_{\delta_{e}}, M_{\delta_{e}}\right]^{\mathrm{T}}
\end{aligned}
$$

BIHS is applied to the parameter estimation problem. The algorithm doesn't estimate all the thirteen parameters at one time, but decompose the identification problem into three subproblems.

$$
\begin{aligned}
& \dot{V}=\left[\begin{array}{llll}
X_{V} & X_{\alpha} & X_{q} & X_{\theta}
\end{array}\right]\left[\begin{array}{c}
V \\
\alpha \\
q \\
\theta
\end{array}\right]+X_{\delta_{e}} \times \delta_{e} \\
& \theta_{1}=\left[X_{v}, X_{\alpha}, X_{q}, X_{\theta}, X_{\delta_{e}}\right]^{\mathrm{T}} \\
& \dot{\alpha}=\left[\begin{array}{llll}
Z_{V} & Z_{\alpha} & Z_{q} & 0
\end{array}\right]\left[\begin{array}{c}
V \\
\alpha \\
q \\
\theta
\end{array}\right]+Z_{\delta_{e}} \times \delta_{e} \\
& \theta_{2}=\left[Z_{v}, Z_{\alpha}, Z_{q}, Z_{\delta_{e}}\right]^{\mathrm{T}} \\
& \dot{q}=\left[\begin{array}{llll}
M_{V} & M_{\alpha} & M_{q} & 0
\end{array}\right]\left[\begin{array}{c}
V \\
\alpha \\
q \\
\theta
\end{array}\right]+M_{\delta_{e}} \times \delta_{e} \\
& \theta_{3}=\left[M_{v}, M_{\alpha}, M_{q}, M_{\delta_{e}}\right]^{\mathrm{T}}
\end{aligned}
$$

\section{B. Parameter Estimation Method}

System input is essential in parameter estimation. In this work, the conventional 3-2-1-1 input is applied, which is the most-used type of input.

The output-error method is a classical approach in aircraft parameter estimation problem. It has several desirable statistical properties [18]. BIHS is used to minimize the output error. $\hat{\theta}$ denotes the estimated parameters. For each given $\hat{\theta}$, the predicted output value and predicted error can be computed by

$$
\begin{aligned}
& \hat{y}(k)=g[\hat{x}(k), u(k), \hat{\theta}] \\
& e(k)=z(k)-\hat{y}(k), k=1 \ldots N
\end{aligned}
$$

The cost function is defined as follows

$$
J=\sum_{i=1}^{N} \sqrt{e_{i}^{2}}
$$

The estimated parameter vector $\hat{\theta}$ is obtained by 


$$
\hat{\theta}=\arg \min _{\hat{\theta}} J(\hat{\theta})
$$

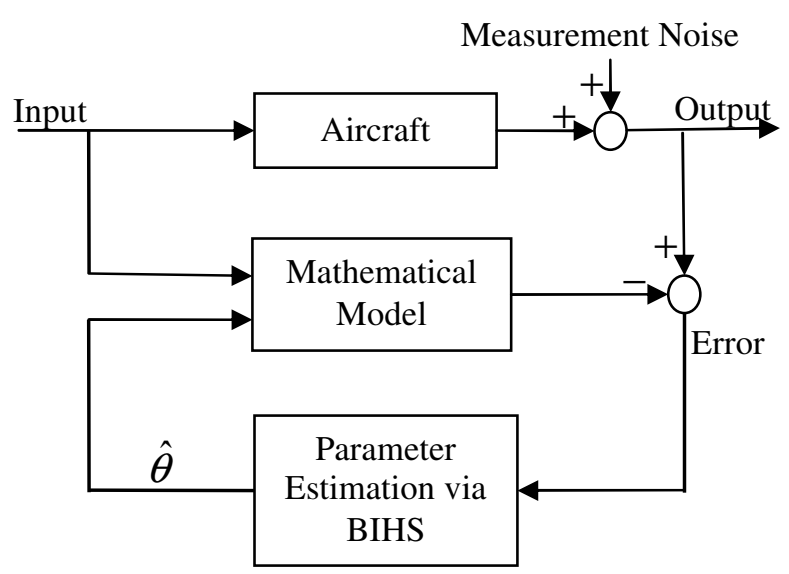

Fig. 2: Procedure of our proposed BISH approach for parameter estimation

\section{SIMULATION RESULTS AND ANALYSIS}

The parameters are estimated by matching the simulation data with the model predicted data. Longitudinal state variables: velocity $V(t)$, attack angle $\alpha(t)$, pitch rate $q(t)$, angle of pitch $\theta(t)$ are obtained from Matlab simulation, and each state variable is contaminated by $30 \mathrm{~dB}$ white Gaussian noise. The sampling period is assumed to be $0.05 \mathrm{~s}$. The simulation lasts for approximately 50s. The 3-2-1-1 elevator input $\delta_{e}$ applied in the simulation is shown in Fig. 3 .

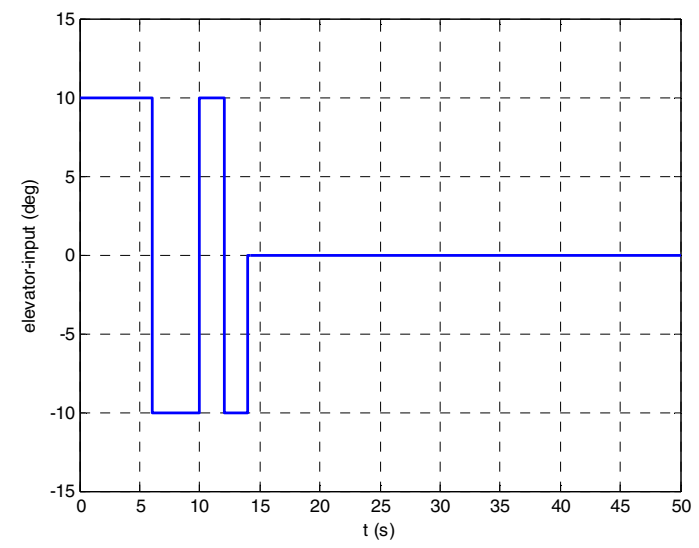

Fig.3.The 3-2-1-1 input

In BIHS, $\mathrm{HMS}=100, \mathrm{HMCR}=0.9, \mathrm{PAR}=0.6$ and the algorithm iterates 5000 times. The measured outputs and the model predicted outputs are shown in Fig.4-Fig.7. As is shown, the output error method via BIHS gives a satisfying longitudinal model of the jet which can precisely predict the output variables.

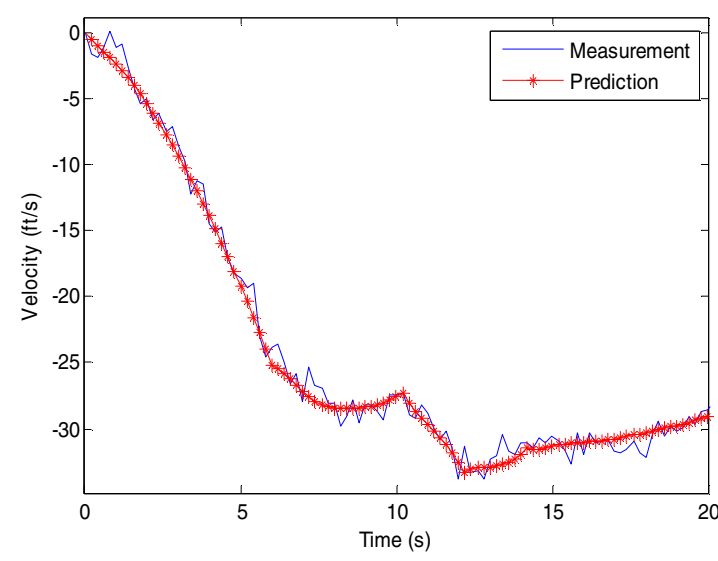

Fig.4. Curves matching of V (t)

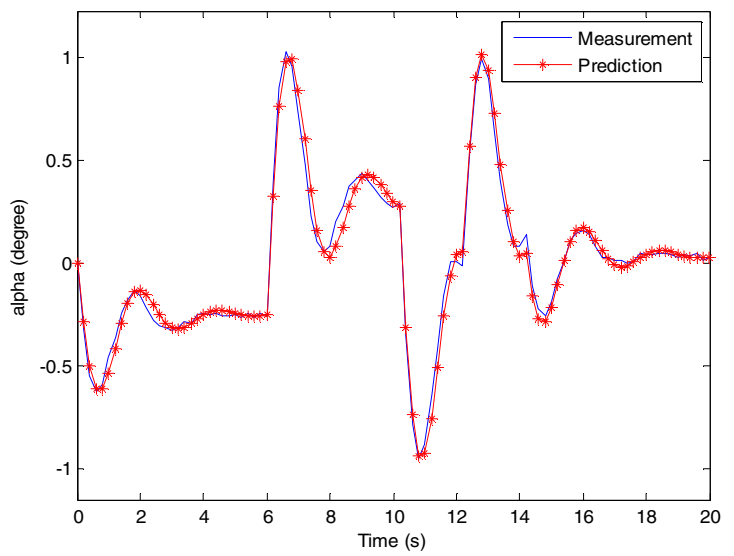

Fig.5. Curves matching of $\alpha(t)$

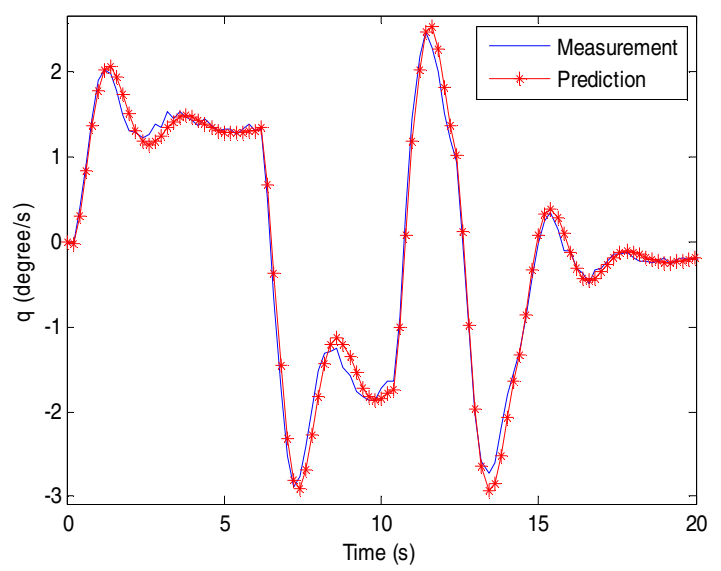

Fig.6. Curves matching of $q(t)$ 


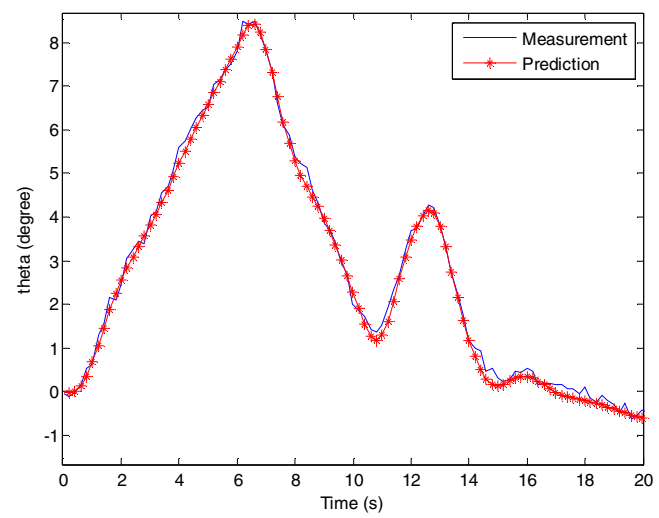

Fig.7. Curves matching of $\theta(t)$

In the traditional ML method, Newton-Raphson is often applied to optimize the maximum likelihood function. However, a good initial value is crucial for Newton-Raphson, or the algorithm may diverge. When the ranges of the unknown parameters are relatively large and a good initial value is not available, the BIHS method seems like a promising alternative to ML. The initial value of NewtonRaphson algorithm is randomly generated in the interval defined in (16), the interval is also taken as the searching range of BIHS,

$$
\begin{gathered}
{[l b, u b]=\left[\text { para }_{\text {ture }}-R, \text { para }_{\text {ture }}+R\right]} \\
R=\text { Range } \times \mid \text { para }_{\text {ture }} \mid
\end{gathered}
$$

where $l b$ and $u b$ are the lower bound and the upper bound of the searching range respectively.

Newton-Raphson algorithm tends to diverge more frequently as the interval gets larger. BIHS is able to search for the unknown parameters in a larger interval, as is shown in Table.1.

TABLE I

COMPARISON BETWEEN BIHS AND NEWTON-RAPHSON

\begin{tabular}{ccc}
\hline \hline \multirow{2}{*}{ Range } & \multicolumn{2}{c}{ Times of divergence in ten runs } \\
\cline { 2 - 3 } & Newton-Raphson & BIHS \\
\hline 0.5 & 0 & 0 \\
0.6 & 1 & 0 \\
0.7 & 1 & 0 \\
0.8 & 3 & 0 \\
0.9 & 4 & 0 \\
1.0 & 5 & 0 \\
1.5 & 8 & 0 \\
\hline \hline
\end{tabular}

BIHS is supposed to converge faster than HS, and have a better ability to escape from local optimum than GHS. To verify its efficiency, we apply BIHS, GHS and HS in the parameter estimation problem. Evolutions curves of four parameters $Z_{V}, Z_{\alpha}, Z_{q}$ and $Z_{\delta_{e}}$ are comparatively shown in Fig.8-Fig.11. And the mean estimation values of the unknown parameters in 50 runs are shown in Table.2.

TABLE II

PARAMETER ESTIMATION RESUltS

\begin{tabular}{cccccc}
\hline \hline & TRUE & Searching & BIHS & HS & GHS \\
Parameters & Value & Range & Method & Method & Method \\
\hline $\boldsymbol{X}_{\boldsymbol{V}}$ & -0.0138 & {$[-0.5,0.5]$} & -0.0132 & -0.0131 & -0.0134 \\
$\boldsymbol{X}_{\boldsymbol{\alpha}}$ & -0.0877 & {$[-0.5,0.5]$} & 0.0569 & 0.0023 & -0.0071 \\
$\boldsymbol{X}_{\boldsymbol{q}}$ & 0.0021 & {$[-0.5,0.5]$} & -0.0595 & -0.054 & -0.0533 \\
$\boldsymbol{X}_{\boldsymbol{\theta}}$ & -0.5618 & {$[-1,1]$} & -0.5614 & -0.5627 & -0.5616 \\
$\boldsymbol{Z}_{\boldsymbol{V}}$ & -0.0079 & {$[-0.5,0.5]$} & -0.0074 & -0.0053 & -0.0067 \\
$\boldsymbol{Z}_{\boldsymbol{\alpha}}$ & -1.0817 & {$[-2,2]$} & -0.9027 & -0.5836 & -0.7208 \\
$\boldsymbol{Z}_{\boldsymbol{q}}$ & 0.9881 & {$[-1,1]$} & 0.9451 & 0.8356 & 0.8647 \\
$\boldsymbol{M}_{\boldsymbol{V}}$ & -0.0055 & {$[-0.5,0.5]$} & -0.0055 & -0.0052 & -0.0054 \\
$\boldsymbol{M}_{\boldsymbol{\alpha}}$ & -7.5943 & {$[-10,10]$} & -7.5787 & -7.4149 & -7.4898 \\
$\boldsymbol{M}_{\boldsymbol{q}}$ & -0.5936 & {$[-1,1]$} & -0.5876 & -0.6149 & -0.5917 \\
$\boldsymbol{X}_{\boldsymbol{\delta} \boldsymbol{e}}$ & -0.208 & {$[-0.5,0.5]$} & -0.1898 & -0.1922 & -0.1932 \\
$\boldsymbol{Z}_{\boldsymbol{\delta} \boldsymbol{e}}$ & -0.1761 & {$[-0.5,0.5]$} & -0.1624 & -0.1353 & -0.1416 \\
$\boldsymbol{M}_{\boldsymbol{\delta} \boldsymbol{e}}$ & -0.1284 & {$[-0.5,0.5]$} & -0.1282 & -0.1193 & -0.1244 \\
\hline \hline
\end{tabular}

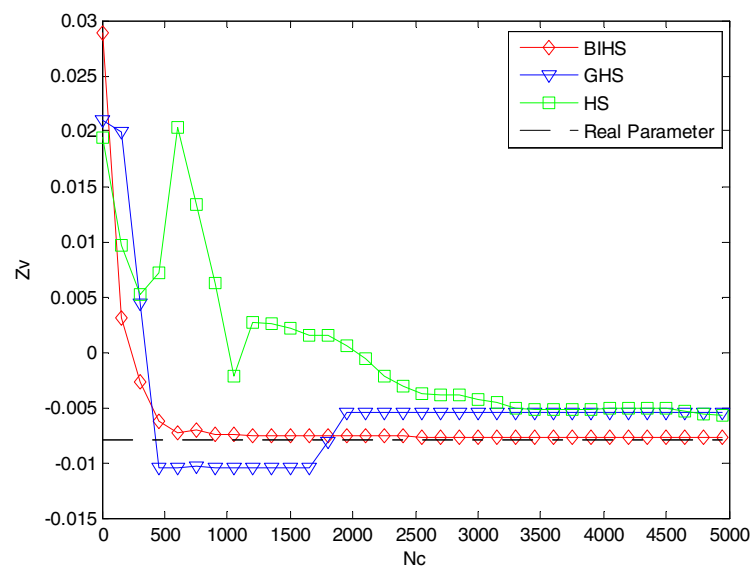

Fig.8. Evolution curves of $Z_{V}$

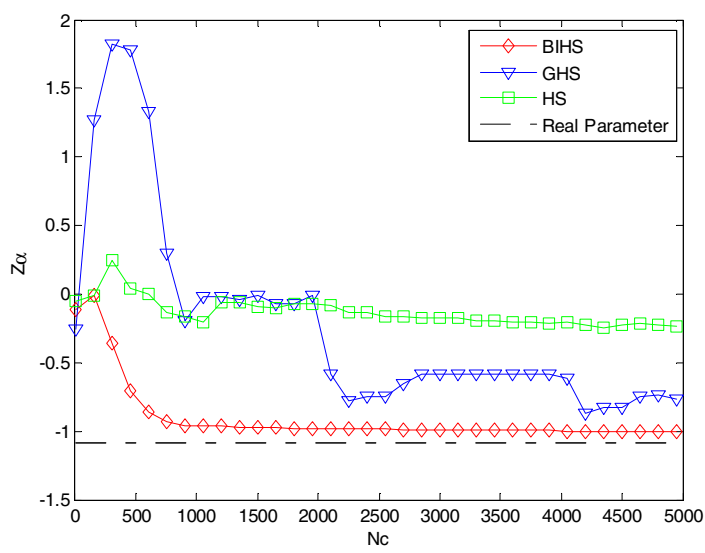

Fig.9. Evolution curves of $Z_{\alpha}$ 


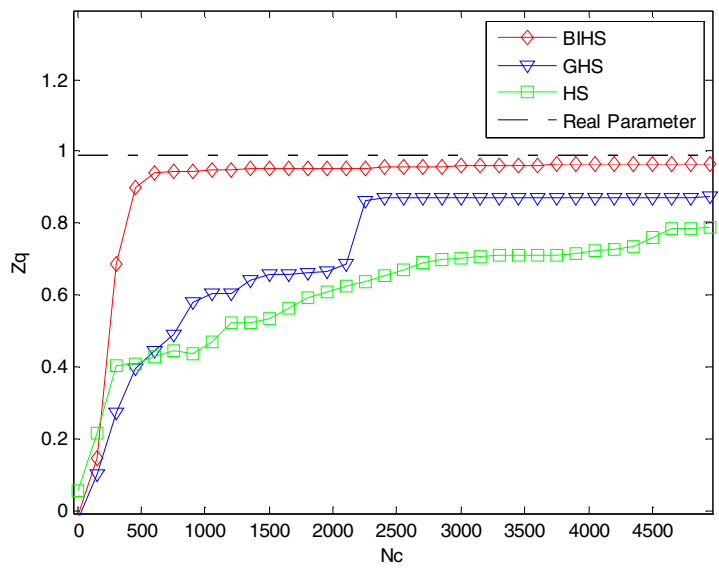

Fig.10. Evolution curves of $Z_{q}$

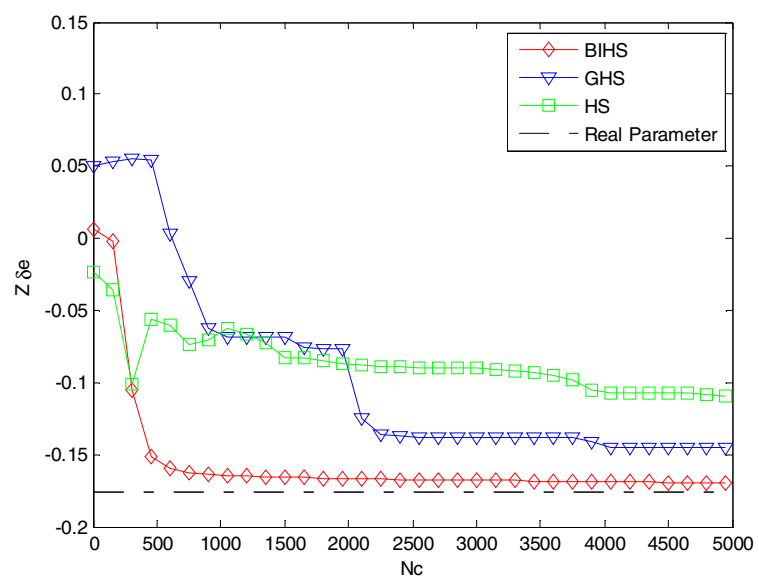

Fig.11. Evolution curves of $Z_{\delta_{e}}$

As is shown in Fig.8-Fig.11, parameters obtained via BIHS method converge to true values faster than that via HS method. In BIHS, parameters move toward the best ones in the flock at a certain possibility. As a result, many aimless searching is avoided. Evolution curves of the cost function are shown in Fig. 12.

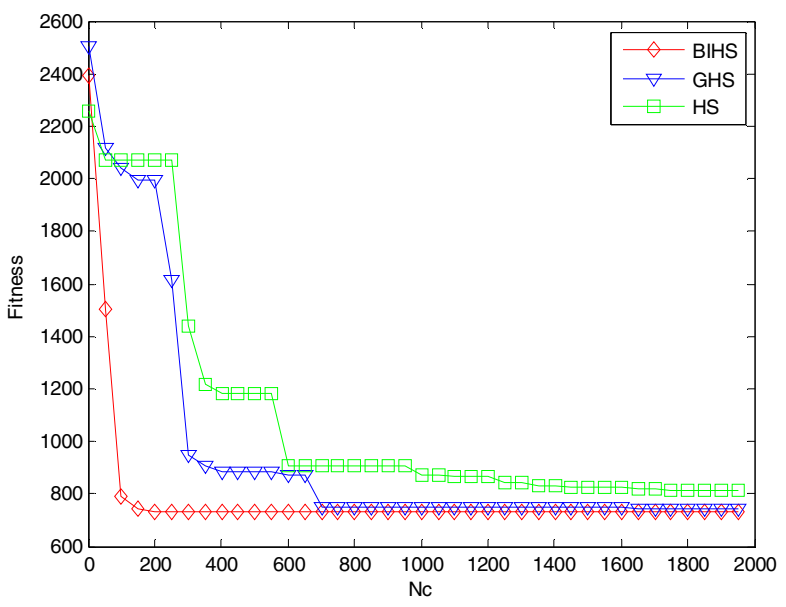

Fig.12. Evolution curves of the cost function for BIHS, GHS and HS

\section{CONCLUSION}

In this paper, we propose a novel method based on BIHS to estimate the unknown parameters in the longitudinal model of F-18 jet. Comparative results show that the modified algorithm converges much faster than the basic HS one.

\section{REFERENCES}

[1] L. Ljung, H. Hjalmarsson, and H. Ohlsson, "Four encounters with system identification," European Journal of Control, vol. 17, no. 5, pp. 449-471, 2011.

[2] L. Ljung, "Issues in system identification," Control Systems, IEEE, vol. 11 , no. 1 , pp. $25-29,1991$

[3] P. Hamel and R. Jategaonkar, "Evolution of flight vehicle system identification," Journal of Aircraft, vol. 33, no. 1, pp. 9-28, 1996.

[4] E. Morelli, "Practical aspects of the equation-error method for aircraft parameter estimation," AIAA Atmospheric Flight Mechanics Conference and Exhibit, Keystone, Colorado, 21 - 24 August, 2006.

[5] T. Söderström and P. Stoica, "Some properties of the output error method," Automatica, vol. 18, no.1, pp. 93-99, 1982.

[6] R. Jategaonkar and E. Plaetschke, "Algorithms for aircraft parameter estimation accounting for process and measurement noise," Journal of Aircraft, vol. 26, no. 4, pp. 360-372, 1989.

[7] C. Picardi and N. Rogano, "Parameter identification of induction motor based on particle swarm optimization," IEEE International Symposium on Power Electronics, Electrical Drives, Automation and Motion, pp. 968973, Taormina, 23-26 May, 2006.

[8] K. Kristinsson and G. Dumont, "System identification and control using genetic algorithms," IEEE Transactions on Systems, Man and Cybernetics, vol. 22, no, 5, pp. 1033-1046, 1992.

[9] I. Jeong and J. Lee, "Adaptive simulated annealing genetic algorithm for system identification," Engineering Applications of Artificial Intelligence, vol. 9, no. 5, pp. 523-532, 1996.

[10]F. Chu, F. Wang, and et al, "A model for parameter estimation of multistage centrifugal compressor and compressor performance analysis using genetic algorithm," Science China Technological Sciences, Vol.55 No.11, pp. 3163-3175, 2012.

[11]C. Xu, H. Duan and F. Liu, "Chaotic artificial bee colony approach to Uninhabited Combat Air Vehicle (UCAV) path planning," Aerospace Science and Technology, vol. 14, no. 8, pp. 535-541, 2010.

[12]H. Duan, S. Shao, B. Su, and et al, "New development thoughts on the bio-inspired intelligence based control for unmanned combat aerial vehicle," Science China Technological Sciences, vol. 53, no. 8, pp. 20252031, 2010.

[13]H. Duan and X. Yu, "Hybrid ant colony optimization using memetic algorithm for traveling salesman problem," in Proceedings of the 2007 IEEE Symposium on Approximate Dynamic Programming and Reinforcement Learning, pp. 92-95, Honolulu ,1-5 April, 2007.

[14]Z. Geem, J. Kim and G. Loganathan, "A new heuristic optimization algorithm: harmony search," Simulation, vol. 76, no. 2, pp: 60-68, 2001.

[15]S. Das, A. Mukhopadhyay, A. Roy, and et al, "Exploratory power of the harmony search algorithm: analysis and improvements for global numerical optimization," IEEE Transactions on Systems, Man, and Cybernetics, Part B: Cybernetics, vol. 41, no, 1, pp.89-106, 2011.

[16]M. Omran and M. Mahdavi, "Global-best harmony search," Applied Mathematics and Computation, vol. 198, no. 2, pp. 643-656, 2008.

[17]C. Reynolds, "Flocks, herds and schools: A distributed behavioral model," Computer Graphics, vol. 21, no. 4, pp. 25-34, 1987.

[18]L. Góes, E. Hemerly, B. Maciel, and et al, "Aircraft parameter estimation using output-error methods," Inverse Problems in Science and Engineering, vol. 14, no. 6, pp. 651-664, 2006. 